\title{
Correlation Analysis between Density and Magnetic Field Low Frequency Fluctuations in Improved Confinement Mode on LHD*)
}

\author{
Wenqing $\mathrm{HU}^{1)}$, Tatsuya KOBAYASHI ${ }^{1,2)}$, Yasuhiro SUZUKI ${ }^{1,2)}$, Mikiro YOSHINUMA ${ }^{2)}$, \\ Tokihiko TOKUZAWA ${ }^{1,2)}$ and Katsumi IDA ${ }^{2)}$ \\ ${ }^{1)}$ The Graduate University for Advanced Studies, SOKENDAI, Toki 509-5292, Japan \\ ${ }^{2)}$ National Institute for Fusion Science, National Institutes of Natural Sciences, Toki 509-5292, Japan
}

(Received 26 November 2020 / Accepted 25 January 2021)

\begin{abstract}
A plasma density fluctuation signal was measured by the recently-installed Beam Emission Spectroscopy (BES). A time-dependent analysis was performed for a discharge in the Large Helical Device (LHD) and compared with the magnetic fluctuation. While the fundamental frequency peak shows a high correlation between the density fluctuation and the magnetic fluctuation, the higher harmonic components have smaller or even negligible correlation. As a possible mechanism that makes the density fluctuation and the magnetic fluctuation different, the relation between the MHD mode and the transport is discussed.
\end{abstract}

(c) 2021 The Japan Society of Plasma Science and Nuclear Fusion Research

Keywords: Large Helical Device, Beam Emission Spectroscopy, L-H transition, magnetohydrodynamic fluctuation, profile saturation

DOI: $10.1585 /$ pfr. 16.2402031

\section{Introduction}

The mode transition phenomena have been reproduced in many different magnetically confined fusion reactors, tokamaks and stellarators [1], while theoretical models have been discussed [2] intending to explain and even provide a reliable scheme to trigger the transition. Examples of outstanding experimental works are listed as follows: the H-mode discharges that not involve the edge localized modes (ELM) are discovered in DIII-D [3] and ASDEX-Upgrade [4]; the high density H-mode is explored in W7-AS [5]; L-H mode transitions triggered by zonal flow are found in EAST [6] and TJ-II [7]; and characteristics of the edge transport barrier are examined in CHS [8].

Magnetohydrodynamic (MHD) mode fluctuation disturbs the confinement of the fusion plasma after the L-H mode transition. Understanding of the structure and mechanics is critical. A representative MHD relaxation phenomenon in tokamak H-mode plasmas is the ELM [9]. Because the heat load to the divertor excited by ELMs is often unacceptably large, a wide variety of schemes to avoid or mitigate ELMs are intensively developed. Similarly, MHD activities after the confinement improvement event are observed in stellarators as well. Previously in the Large Helical Device (LHD), a soft X-ray emission diagnostics array was used to examine the spatial structures of the MHD fluctuation [10]. As the soft X-ray emission diagnostics can only provide the line integrated quantity, another local and multichannel diagnosis with high spatial resolution is necessary for further understanding.

author's e-mail: hu.wenqing@nifs.ac.jp

*) This article is based on the presentation at the 29th International Toki Conference on Plasma and Fusion Research (ITC29).
Beam Emission Spectroscopy is a well-developed diagnostic tool that can provide simultaneous characteristic measurements of the density fluctuation and has been installed not only in tokamaks [11-15] but also in stellarators [16-20]. Recently, a BES measurement system was installed onto LHD and a routine measurement was begun in the 2019 LHD experiment campaign [20].

By analyzing the BES signal and comparing it with other measurements, we found that multiple spectral peaks with a regular frequency spacing are excited in both the density fluctuation and the magnetic fluctuation after the L-H confinement mode transition. The fluctuation at the fundamental frequency has a high correlation between the density fluctuation and the magnetic fluctuation. However, the density and the magnetic field behave almost independently at the higher harmonic frequencies. This observation implies that the mode distorts differently in the plasma density and the magnetic field. In this paper, we briefly introduce the BES in LHD and then the methodology in detail. Next, we discuss the analysis results and problems for future exploration. The last section is dedicated to the summary.

\section{BES Measurement}

BES uses the Doppler shifted $\mathrm{H}_{\alpha}$ emission to measure the density fluctuation in plasma. An $8 \times 8$ array of optical elements is installed onto LHD through the port 6-O. Lines of sight cover from the mid-radius $\left(r_{\mathrm{eff}} / a_{99} \approx 0.5\right)$ to the edge $\left(r_{\text {eff }} / a_{99} \approx 1.0\right)$ as shown in Fig. 1 as $\square$. The dasheddot line is the border of $a_{99}$ which contains $99 \%$ of the plasma stored energy while the color bar value represents the $r_{\text {eff }}$, the effective minor radius [21]. The angle between 


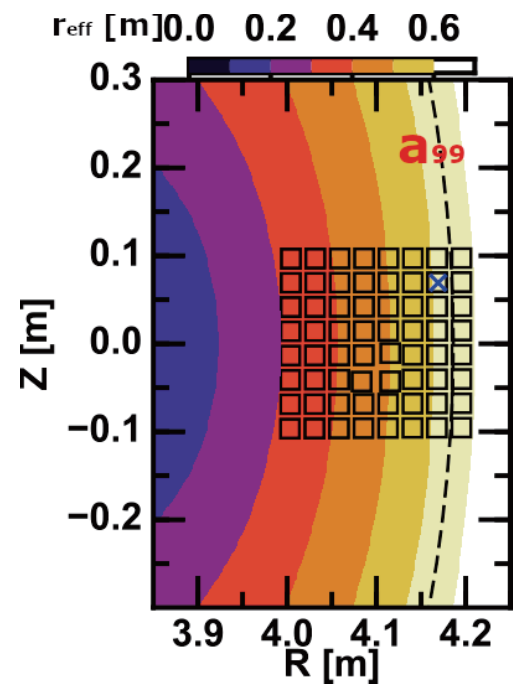

Fig. 1 Poloidal cross section of the BES Lines of Sight (LoS) shown as $\square$ in LHD. $\times$ in the figure is the channel used for this analysis.
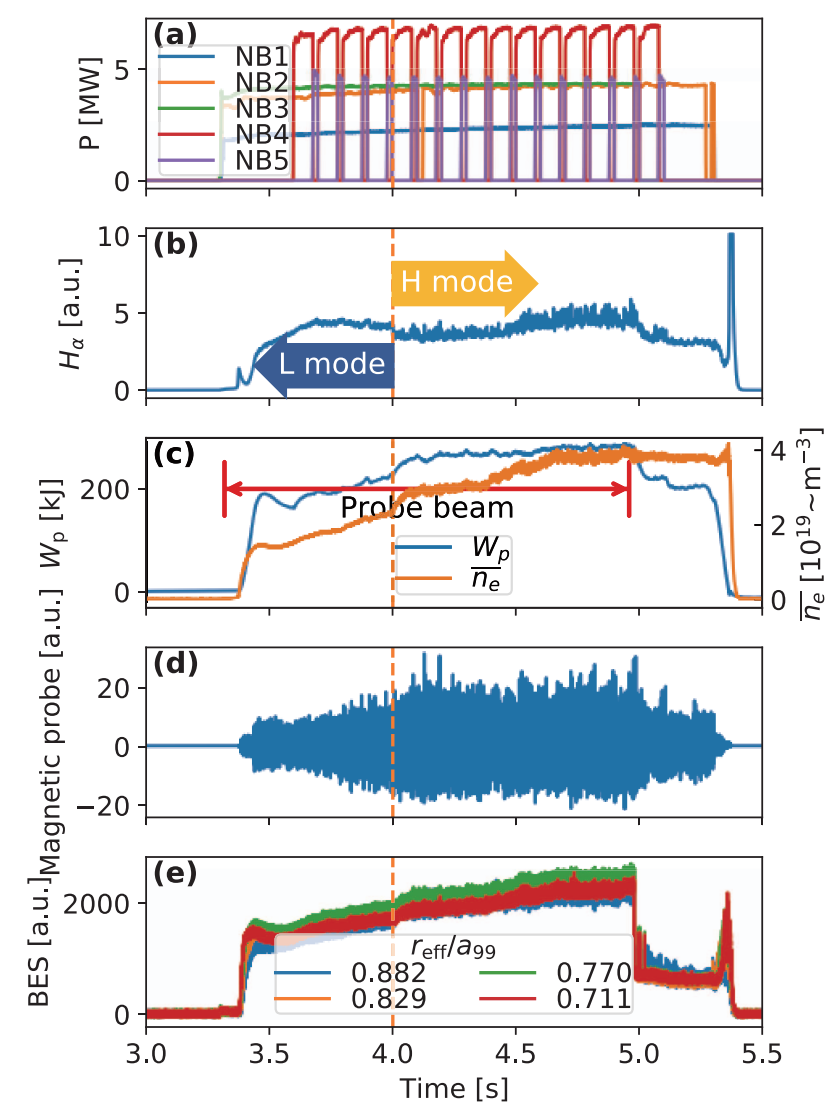

Fig. 2 Discharge condition and parameters: (a) NBI input power, (b) $\mathrm{H}_{\alpha}$ emission, (c) plasma stored energy $W_{\mathrm{p}}$ and line averaged electron density $\overline{n_{\mathrm{e}}}$, (d) magnetic probe signal, (e) BES signals.

the lines of sight and the neutral beam injection (NBI) axis is set to be $150.6^{\circ}-153.6^{\circ}$. A heating neutral beam with the acceleration energy of $165 \mathrm{keV}$ (NBI\#3) is used as the probe beam [20]. The shifted $\mathrm{H}_{\alpha}$ emission is extracted

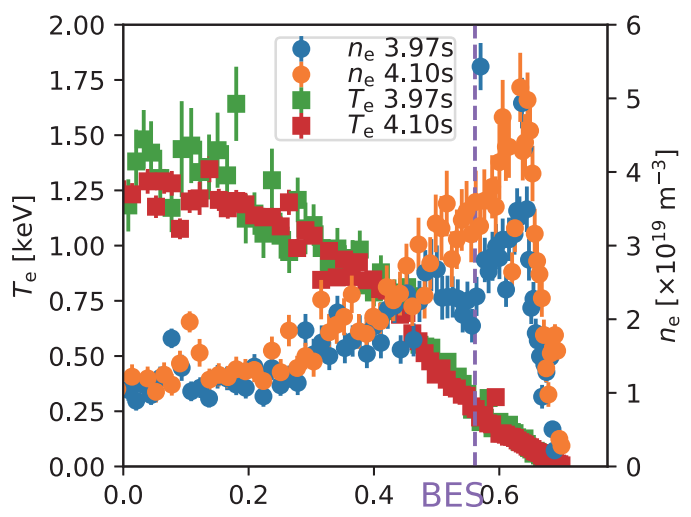

Fig. 3 Electron density $n_{\mathrm{e}}$ and temperature $T_{\mathrm{e}}$ before $(3.9669 \mathrm{~s})$ and after $(4.1003 \mathrm{~s})$ the L-H mode transition.

using a rotatable filter (CLF-400 BUNKOUKEIKI) [22]. The extracted light is led to an avalanche photo diode camera (APDCAM-10 g Fusion Instrument Kft.) operated at a sampling frequency of $200 \mathrm{kHz}$. Since frequencies of our target fluctuations are less than $10 \mathrm{kHz}$, the BES signal is down-sampled to $100 \mathrm{kHz}$ in this research. Therefore, the Nyquist frequency of the BES signal is $50 \mathrm{kHz}$.

Time evolution of the discharge parameters and measured magnetic probe and BES signals of the shot \#156774 is shown in Fig. 2. The first row shows the NBI pulses with NBI\#3 as probe beam of BES on from 3.30 to $4.97 \mathrm{~s}$. The second row is the $\mathrm{H}_{\alpha}$ signal with a spontaneous drop at $4.0 \mathrm{~s}$ indicating a L-H mode transition. The third row shows the line averaged density $\overline{n_{\mathrm{e}}}$ and the diamagnetic plasma stored energy $W_{\mathrm{p}}$. The plasma gains a higher line averaged density after the mode transition and presents an increase in the plasma stored energy. The fourth row is a magnetic probe signal with rougher spikes after mode transition indicating more significant magnetic fluctuation. The last row is the BES signal. Since NBI\#3 as probe beam shutdown at $4.97 \mathrm{~s}$, the BES signal after $4.97 \mathrm{~s}$ is considered to be the background emissions.

Electron density and temperature before and after L-H mode transition is shown in Fig. 3. At the edge region, the electron density increases while temperature remains the same. As a result, the electron pressure increases accordingly. This is the typical property of the L-H transition in LHD [23]. A similar confinement improvement in the edge density transport channel is reported in Heliotron $\mathrm{J}[24,25]$.

\section{Analysis Results}

\subsection{Frequency-based analysis: cross coherence}

A spectrogram was created using the BES and magnetic probe signals by calculating the power spectral density through discrete Fourier transform with 1000 data per segment and 500 data shift. The 1000 data per segment makes the frequency resolution of the spectrogram and the cross coherence become $0.1 \mathrm{kHz}$. A larger time window 

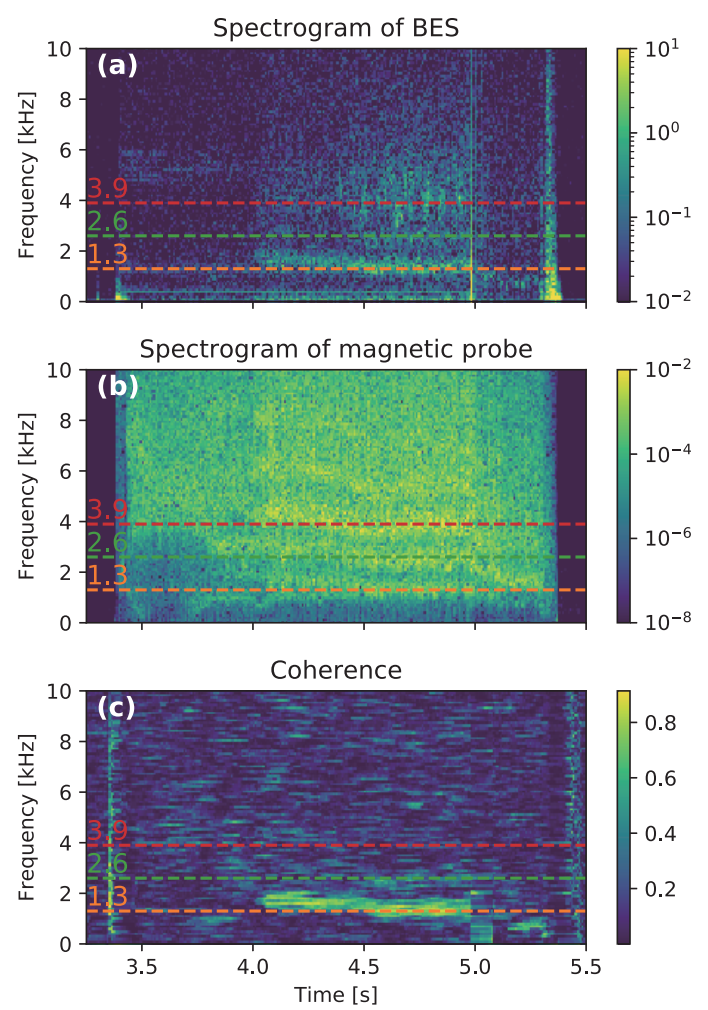

Fig. 4 Spectrogram of (a) BES and (b) magnetic probe signal and (c) coherence.

increases the noise level in the coherence due to the insufficient ensemble number. On the contrary, the coherence result was qualitatively the same when the time window is halved. The squared cross coherence is calculated using the following equation:

$$
\gamma^{2}=\frac{\left|S_{\tilde{n}, \tilde{B}}(\omega)\right|^{2}}{S_{\tilde{n}}(\omega) S_{\tilde{B}}(\omega)},
$$

where $S_{\tilde{n} \tilde{B}}$ is the cross-spectrum while $S_{\tilde{n}}$ and $S_{\tilde{B}}$ are the auto-spectrum of the BES and magnetic probe signal, respectively. Time averaging of the cross spectrum is performed in 6 successive windows.

Time evolution of the spectrogram of BES and magnetic probe, and the squared cross-coherence of the two is shown in Fig. 4. In this data, the BES measures the density fluctuation at $r_{\text {eff }} / a_{99}=0.85 \pm 0.046$. The chosen BES channel has the highest signal to noise ratio. To confirm the robustness of the analysis results in this manuscript, we also attempted to make the same analysis by using the BES signals at different channels nearby and the different magnetic probe signals in the toroidal array. The results obtained had basically the similar characteristics. Although both BES and magnetic probe show peaks in frequency between approximately $1-6 \mathrm{kHz}$, the squared cross coherence only appears to be high in lower frequency. The same phenomenon can be observed in the long time average power spectral density shown in Fig. 5. Here, it is clearer that both power spectral densities of the BES and

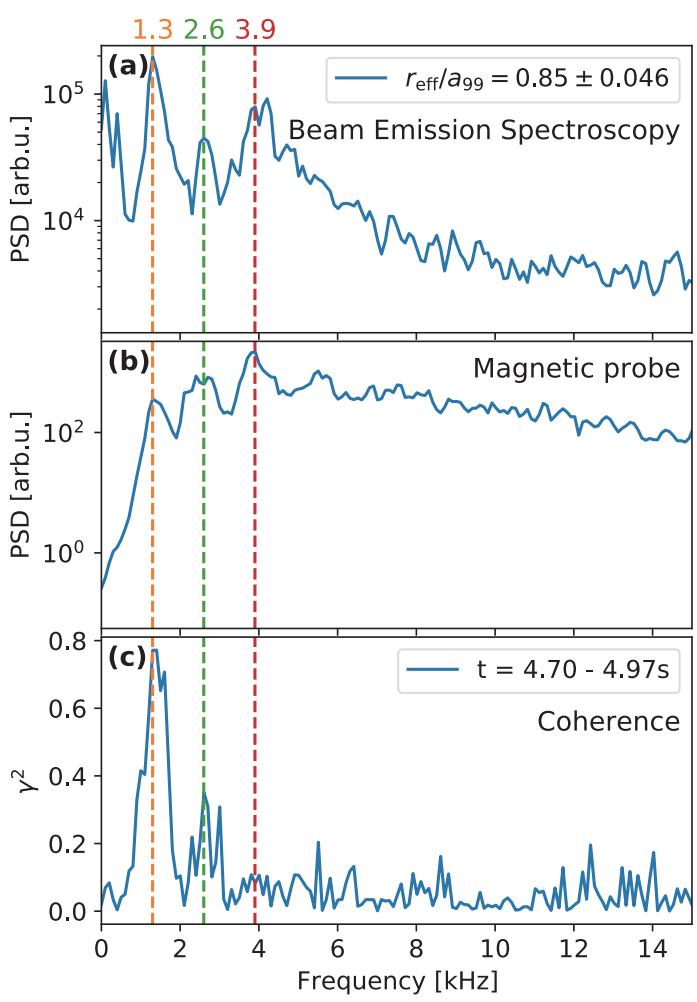

Fig. 5 Time-averaged power spectral density of (a) BES and (b) magnetic probe signal and (c) coherence.

the magnetic probe have signal peaks at $1.3 \mathrm{kHz}, 2.6 \mathrm{kHz}$ and $3.9 \mathrm{kHz}$, but only at $1.3 \mathrm{kHz}$ does significant squared cross coherence appear. Overall, the spectral peak of the BES signal at $f=3.9 \mathrm{kHz}$ is broader than other peaks. As can be seen in Fig. 4 (a), time evolution of the spectral peak value around $f=3.9 \mathrm{kHz}$ behaves intermittently, unlike the power spectrum of the magnetic probe signal. This intermittent behavior of the BES spectrum may provide an additional peak at $f=4.1 \mathrm{kHz}$, which likely has the same fluctuation property with that of the peak at $f=3.9 \mathrm{kHz}$. In addition, the cross-coherence between the magnetic probe signal and a $\mathrm{CO}_{2}$ interferometer signal is also confirmed to have a qualitatively similar characteristic as that of the BES signal. A low cross-coherence in the higher harmonics corresponds to the phase difference between the magnetic probe signal and the BES signal varying randomly in time.

According to the phase analysis of the toroidal and poloidal magnetic probe arrays and the BES spatiotemporal analysis, the modes are considered to be coherent MHD modes excited at the plasma edge. After the L-H transition, the edge plasma boundary is slightly extended and new rational surfaces come into the plasma volume. As a result, coherent MHD modes are excited and likely limit further increase of the edge density. In order to discuss the role of the MHD modes on the density profile saturation, time dependent properties of the density fluctuation are compared to those of the magnetic fluctuation in detail in the next 


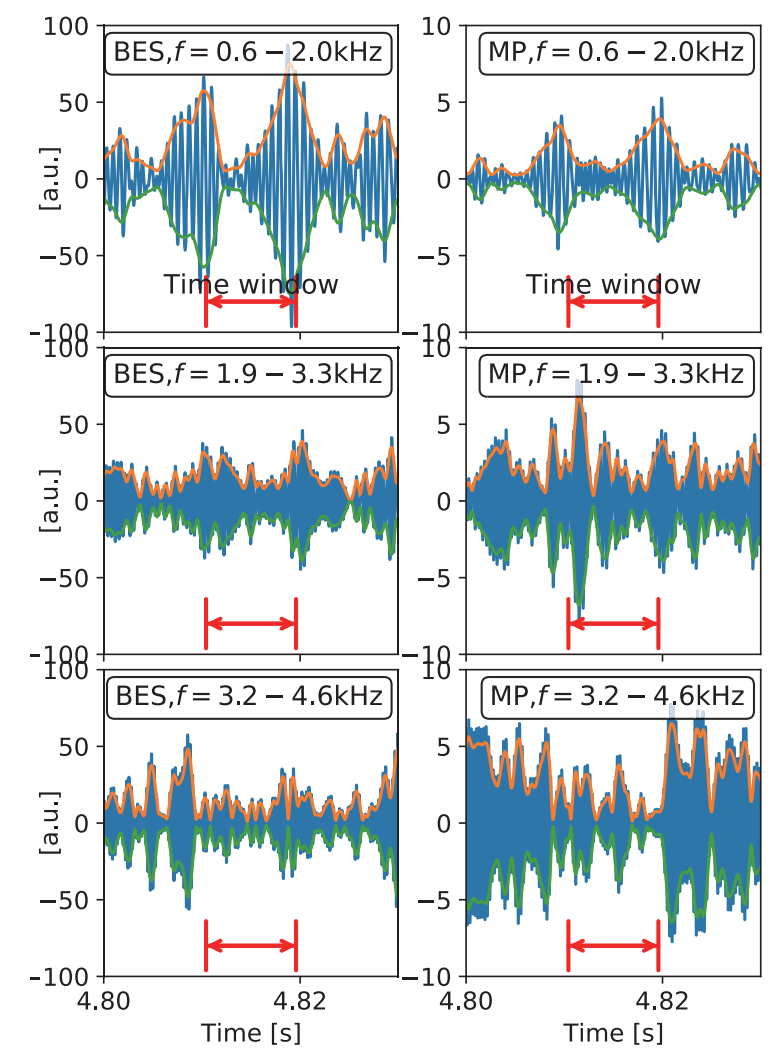

Fig. 6 Band-pass-filtered signals of BES and magnetic probe and the envelopes.

subsection.

\subsection{Time-based analysis: cross-correlation}

To examine the phenomena further, a correlation analysis is conducted on the BES and the magnetic probe signal. We used a band-pass filter to extract the signal in the $1.3,2.6$ and $3.9 \mathrm{kHz}$ frequency bands, respectively. The band width of the filter is set to be $0.7 \mathrm{kHz}$. The envelope is evaluated for each frequency band by calculating the root mean square of the filtered signal and its Hilbert transform. Another low-pass filter with the lower border of the prior band pass filter range as the cut-off frequency is operated afterwards to eliminate the high-frequency noise induced by the envelope calculation. As an example, Fig. 6 shows the time evolution of the band-pass-filtered signals of BES and magnetic probe as well as their envelopes between $4.80-4.83 \mathrm{~s}$. The envelopes of the BES signal and the magnetic probe signal behave similarly at the fundamental frequency band. While, they evolve nearly independently in the higher harmonic frequency bands. The length of the time window used for the Fourier spectral analysis in the previous subsection is overlaid on each figure panel. It is found that the length of the time window is larger than the typical time period of the envelope evolution for the higher harmonic components. Therefore, the intermittency of the signal has been reflected on the spectral peak widths in Fig. 5.

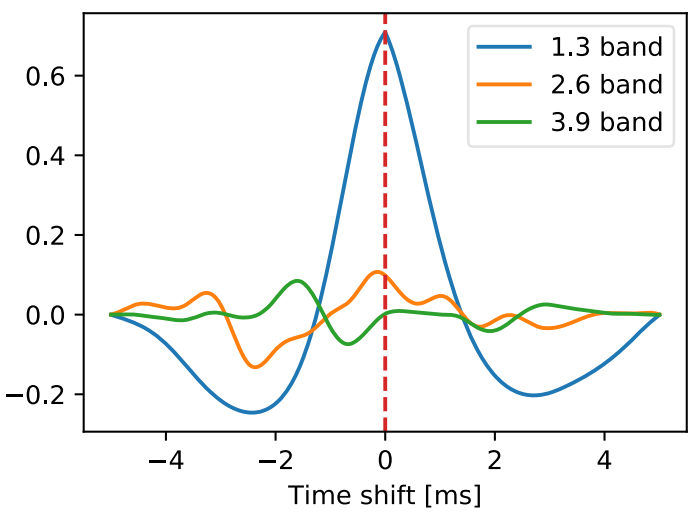

Fig. 7 Cross correlation between BES and magnetic probe signal at $1.3 \mathrm{kHz}, 2.6 \mathrm{kHz}$ and $3.9 \mathrm{kHz}$.

Cross-correlation between the BES signal and the magnetic probe signal is calculated for each frequency band. Each envelope was subtracted by its average to leave only the alternating component. The correlation is then calculated according to the following definition:

$$
C_{\hat{n} \hat{B}}(\tau)=\frac{1}{T} \int_{-\frac{T}{2}}^{\frac{T}{2}} \hat{n}(t+\tau) \hat{\dot{B}}(t) \mathrm{d} t
$$

where, the hat indicates the signal envelope. The result is shown in Fig. 7. Only the mode at $1.3 \mathrm{kHz}$ shows a significant correlation $(>0.6)$ with zero time shift. Zero time shift at $1.3 \mathrm{kHz}$ indicates that density fluctuation energy and magnetic fluctuation energy both increase/decrease simultaneously.

\section{Discussion}

We learned several facts from the two analyses. The first analysis not only proved that the BES signal is legitimate by showing coherence with the magnetic probe signal, but also provided new information that there exists higher-frequency density fluctuations whose characteristics do not match that of magnetic fluctuations. The second analysis confirmed the unmatched signal in time base by showing the low correlation between BES and magnetic probe signal at higher frequency bands. Those two results correspond each other. The second analysis also suggests no time-delay between the density fluctuation energy and the magnetic fluctuation energy.

We made a hypothesis based on the analysis result. At the base frequency, the density fluctuation and magnetic fluctuation has high correlation. Meanwhile, for higher frequency, these two have smaller or even negligible correlation. The higher harmonic have different properties of the fundamental mode. The present observation implies that the density and magnetic field fluctuate differently. In a simplest view of the density fluctuation excited by the MHD activity, the density profile is tied to the magnetic fluctuation such that the density fluctuation should be identical to the magnetic fluctuation. However, that is not the 
case here. A possible factor that makes the density fluctuation and the magnetic fluctuation different is the transport. The particle transport can be modulated by the MHD fluctuation on the magnetic surface, and can couple to the density profile. The coupling between the MHD mode and the transport can be a key player that determines the saturated density profile in the $\mathrm{H}$-mode and therefore determines the achievable beta value. In order to further examine this hypothesis of the MHD-particle transport coupling, the following steps need to be taken. First, in order to quantitatively affirm the present observation, intensive cross-check using different diagnostics systems is necessary to be performed. Apparently, different measurement principle and configuration between the on-vessel magnetic probe and the BES can affect the value of correlation. In addition, correlation analysis with respect to the electron cyclotron emission (ECE) diagnostic would also be worthwhile for discussing the MHD-thermal transport coupling. Another possible step is direct investigation of the particle flux both in experiment and simulation.

\section{Summary}

Characteristics of the density fluctuation and the magnetic fluctuation are compared through the BES and magnetic probe signals. For the frequency-based analysis, we discovered that the two signals only have high coherence at the lowest frequency peak. For the time-based analysis, we calculated the correlation for each frequency band and only the low frequency fluctuation component showed significant correlation. The observed mode had different density and magnetic field fluctuations in the higher harmonic mode. As a possible mechanism that made the density fluctuation and the magnetic fluctuation different, the relation between the MHD mode and the transport was discussed.

\section{Acknowledgments}

The authors thank all the members of the LHD Experiment Group for their assistance, and S. Ohdachi and Y. Takemura for useful discussions. This work is partly supported by the National Institute for Fusion Science Grants (ULHH033) and by the Grant-in-Aid for Scientific Research of JSPS (15H02336, 17K14898). This work was partially supported by "PLADyS", JSPS Core-to-Core Program, A. Advanced Research Networks.

[1] F. Wagner, Plasma Phys. Control. Fusion 49, B1 (2007).

[2] J.W. Connor et al., Plasma Phys. Control. Fusion 42, R1 (2000).

[3] K.H. Burrell et al., Plasma Phys. Control. Fusion 44, A253 (2002).

[4] W. Suttrop et al., Plasma Phys. Control. Fusion 45, 1399 (2003).

[5] K. McCormick et al., Phys. Rev. Lett. 89, 015001 (2001).

[6] P. Manz et al., Phys. Plasmas 19, 072311 (2012).

[7] T. Estrada et al., Nucl. Fusion 55, 063005 (2015).

[8] T. Minami et al., J. Phys. Conf. Ser. 123, 012006 (2008).

[9] H. Zohm, Plasma Phys. Control. Fusion 38, 105 (1996).

[10] F. Watanabe et al., Plasma Phys. Control. Fusion 48, A201 (2006).

[11] R.J. Fonck, Rev. Sci. Instrum. 61, 3487 (1990).

[12] G.R. McKee et al., Rev. Sci. Instrum. 74, 2014 (2003).

[13] A.R. Field et al., Rev. Sci. Instrum. 83, 013508 (2012).

[14] M. Lampert et al., Rev. Sci. Instrum. 86, 073501 (2015).

[15] W. Mandl et al., Plasma Phys. Control. Fusion 35, 1373 (1993).

[16] T. Oishi et al., Rev. Sci. Instrum. 75, 4118 (2004).

[17] S. Kado et al., Rev. Sci. Instrum. 81, 10 D720 (2010).

[18] M. Ono et al., Plasma Fusion Res. 11, 1402115 (2016).

[19] S. Kobayashi et al., Rev. Sci. Instrum. 81, 10D726 (2010).

[20] T. Kobayashi et al., Plasma Phys. Control. Fusion 62, 125011 (2020).

[21] C. Suzuki et al., Plasma Phys. Control. Fusion 55, 014016 (2012).

[22] V. Olevskaia et al., Plasma Fusion Res. 14, 1305118 (2019).

[23] K. Toi et al., Fusion Sci. Technol. 58, 61 (2010).

[24] F. Sano et al., Nucl. Fusion 45, 1557 (2005).

[25] S. Kobayashi et al., 26th IAEA Fusion Energy ference IAEA CN-234, EX/P8-17 (2017). 